\title{
Mejoras en la gestión ambiental del hotel Iberostar Ordoño del destino turístico de Gibara, Cuba
}

\section{Improvements in environmental management of the Iberostar Ordoño hotel in the tourist destination of Gibara, Cuba}

Dr. C. Migdely Barbarita Ochoa Avila. ${ }^{1}$, Lic. Dainis Leyva Driggs. ${ }^{2}$

\begin{abstract}
The Iberostar Ordoño Hotel has a notable influence on the environment of Gibara, Cuba's touristic destination, which demands an integral environmental management. The investigation had as objective to develop a procedure that incorporates the environmental management in a traverse way to the rest of the processes of the hotel, focused by strategic, operative and support component, that favors the improvement of the environment, to foment the good operation of the installation, to execute legislations and at the same time to improve the administration of human, material, economic and technological resources, as well as the image perceived by the clients. Theoretical, empiric and statistical methods were used, as well as instruments for the gathering, prosecution, analysis and interpretation of the indicators associated to each component. The procedure consists of four phases and thirteen steps, and it was applied partially until the phase II, item 7. The main practical contribution of the investigation was the realization of a diagnosis to each component, as well as the creation of an environmental management's program with strategic actions to achieve the transformation of the identified problems. The results of the investigation in the Iberostar Ordoño Hotel not just contribute to the improvement of the environment, but also to the execution of the objectives 4, 6, 7, 8, 11 and 12 of the Calendar 2030 for the Sustainable Development and to the Tasks 4, 8, 10 and 11 of the State of Cuba Plan up to the 2050 for the confrontation to the climatic change.
\end{abstract}

Key Words: Environmental Management, Environmental Management in City Hotels, Components of The Environmental Management, Procedure for The Environmental Management

\footnotetext{
${ }^{1}$ Profesora Titular Departamento de Turismo Universidad de Holguín, Cuba, E-mail: migdely@uho.edu.cu

${ }^{2}$ Licenciada en Turismo, Agencia de Viajes TRANSTUR Holguín, Cuba, E-mail: dai.ld@ nauta.cu
} 


\section{Resumen}

El Hotel Iberostar Ordoño ejerce notable influencia sobre el medio ambiente de Gibara, Destino turístico de Cuba, lo que demanda una gestión ambiental integral. La investigación tuvo como objetivo desarrollar un procedimiento que incorpore la gestión ambiental de forma transversal al resto de los procesos del hotel, enfocado por componente estratégico, operativo y de apoyo, que favorezca la mejora del medio ambiente, para fomentar el buen funcionamiento de la instalación, cumplimentar legislaciones y a su vez mejorar la gestión de recursos humanos, materiales, económicos y tecnológicos, así como la imagen percibida por los clientes. Se utilizaron métodos teóricos, empíricos y estadísticos, así como instrumentos para la recolección, procesamiento, análisis e interpretación de los indicadores asociados a cada componente. El procedimiento consta de cuatro fases y trece pasos, y fue aplicado parcialmente hasta la fase II, paso 7. El principal aporte práctico de la investigación fue la realización del diagnóstico a cada componente, así como la propuesta del programa de gestión ambiental con acciones estratégicas para lograr la transformación de las problemáticas identificadas. Los resultados de la investigación en el Hotel Iberostar Ordoño, contribuyen no solo a la mejora del medio ambiente, sino además al cumplimiento de los objetivos 4, 6, 7, 8, 11 y 12 de la Agenda 2030 para el Desarrollo Sostenible y a las Tareas 4, 8, 10 y 11 del Plan de Estado de Cuba hasta el 2050 para el enfrentamiento al cambio climático.

Palabras Clave: Gestión Ambiental, Gestión Ambiental En Hoteles de Ciudad, Componentes de la Gestión Ambiental, Procedimiento para la Gestión Ambiental

\section{Introducción}

La gestión ambiental es un proceso de relevancia en las organizaciones. Isaac Godínez (2012), considera que es un concepto integrador superior al del manejo ambiental: directrices, lineamientos y políticas formuladas desde los entes rectores, que median en la implementación. Para Atencio Zayas (2015) es un campo temático amplio que hace énfasis en la aplicación de mecanismos normativos y técnicas encaminadas a fortalecer la capacidad humana e institucional para abordar eficazmente las cuestiones ambientales. De significación resulta la definición de la ISO 14001 (2015), la cual establece que la gestión ambiental es un proceso que permite fomentar y llevar a cabo la política ambiental y los objetivos marcados por la organización para llevar a cabo un adecuado desempeño ambiental.

Cuba destaca entre sus tendencias actuales la identificación cada vez mayor de sus interrelaciones con el medio ambiente, lo cual se evidencia a través de leyes y regulaciones, además de la participación en proyectos y eventos que se realizan por entidades reguladoras como el Ministerio de Ciencia, Tecnología y Medio Ambiente (CITMA). Cada día se trabaja para lograr un desarrollo turístico ambiental sano, enfocándose en la creación de estrategias ambientales que gestionen el medio ambiente de manera eficiente, pero todavía existen locales en el sector que presentan faltas en cuanto a esta gestión, provocando un mal desempeño ambiental de la localidad en que radican. 
Al respecto se han aplicado metodologías de gestión ambiental en organizaciones turísticas cubanas (Campbell Margenat (2009), Lorenzo Linares (2011), González Abreu (2015), Paz Ramírez (2015), Azze Alonso (2016), Martínez Rodríguez (2017)), basadas en fases y pasos que hacen énfasis en la planificación de acciones transformadoras con respecto a las problemáticas ambientales identificadas en los procesos, así como en la implantación de las acciones y la auditoría para verificar la eficacia del proceso.

Gibara, municipio ubicado al norte de la provincia de Holguín, fue lanzado como nuevo Destino turístico en 2017, pero el turismo de esta zona se ha visto afectado por problemas ambientales que amenazan y atentan contra la buena imagen del destino por parte de los visitantes. Fenómenos como la contaminación (residuales líquidos, sólidos, emisiones a la atmósfera, sónica, etc.) constituyen los principales problemas ambientales vigentes en el municipio, que al no ser erradicados pueden llegar a traer serias consecuencias para la actividad de explotación turística y su vínculo con el desarrollo económico y social.

Una de las organizaciones turísticas más importantes del Destino es el Hotel Iberostar Ordoño, perteneciente a la cadena Iberostar Hotels \& Resorts y al Grupo Hotelero Cubanacán, ejerce una notable influencia en el medio ambiente de este destino. Es un hotel cuatro estrellas, de 27 habitaciones, ubicado muy cerca de la superficie costera, por lo que recibe afectaciones ante los efectos del cambio climático. La instalación en sus inicios carecía de una Estrategia Ambiental propia, solo se contaba con una Estrategia Ambiental de la Empresa en general, sin embargo actualmente la cadena Iberostar Hotels \& Resorts, ha fortalecido este aspecto en sus instalaciones a nivel de país, por lo que ha realizado transformaciones favorables con respecto al desempeño ambiental, entre ellas figuran: el establecer el cargo de responsable del medio ambiente y sus funciones, la actualización de la documentación y recopilación de aspectos legales, normas y reconocimientos ambientales. Estas han sido trabajadas en el Hotel Iberostar Ordoño, además de programar diversas acciones relacionadas con el cumplimiento del Plan de Estado Tarea Vida, documento conformado a nivel de país para enfrentar el cambio climático.

A pesar de los avances anteriores, la revisión ambiental preliminar del Hotel Iberostar Ordoño realizada en el año 2018 detectó que existían problemas ambientales que se refieren a factores estratégicos, contextuales y organizativos, que incidían en el cumplimiento de su misión social. A partir de lo expuesto anteriormente, pero detectó como problema: Las insuficiencias en la gestión ambiental, limitan la mejora del medio ambiente en el Hotel Iberostar Ordoño.

\section{Desarrollo}

El objetivo de este trabajo es desarrollar un procedimiento para la gestión ambiental integral en el Hotel Iberostar Ordoño, que contribuya a la mejora del medio ambiente.

Los objetivos específicos planteados fueron:

1. Elaborar los fundamentos teóricos-prácticos referenciales que sustentan la gestión ambiental en hoteles. 
2. Diseñar el Procedimiento para la gestión ambiental integral en el Hotel Iberostar Ordoño.

3. Aplicar de forma parcial el Procedimiento para la gestión ambiental integral en el Hotel Iberostar Ordoño.

4. Valorar el procedimiento propuesto a través del criterio de especialistas.

La metodología aplicada fue la investigación cualitativa a través de los métodos:

\section{Métodos teóricos}

- Análisis-síntesis: en la fundamentación teórica de la investigación, revisión de documentos normativos del Ministerio del Turismo, Ministerio de Ciencia Tecnología y Medio Ambiente (CITMA), familias de normas cubanas NC ISO 14001 sobre el Sistema de Gestión Ambiental. De utilidad para resumir las principales premisas teórico-metodológicas de la relación medio ambiente y turismo, para sintetizar aquellos aspectos positivos y negativos relacionados con la gestión ambiental en hoteles de ciudad, que influyen en el desempeño ambiental de la instalación turística.

- Inductivo-deductivo: en el establecimiento de nexos y relaciones entre los conceptos de gestión ambiental, procedimientos propuestos por otros autores, revisión bibliográfica y documentación especializada, así como para conocer los problemas ambientales actuales que presenta el Hotel Iberostar Ordoño.

\section{Métodos empíricos}

- Observación participante: para identificar y diagnosticar los problemas ambientales por componentes que están presente en la instalación hotelera.

- La entrevista y encuesta: a directivos, trabajadores y clientes del hotel para diagnosticar la gestión ambiental por componente estratégico, operativo y de apoyo.

- Consulta a especialistas: empleada para valorar el procedimiento propuesto a partir de los presupuestos relacionados con el estado actual de la gestión ambiental en hoteles y su contextualización para el Hotel Iberostar Ordoño.

\section{Métodos estadísticos}

- Estadística descriptiva: para la recopilación, presentación e interpretación de los resultados obtenidos.

\section{Resultados}

\section{Particularidades de la gestión ambiental en hoteles de ciudad}

Los hoteles de ciudad, característicos por su importancia cultural y artística o por su desarrollo industrial - económico, al igual que el resto de las instalaciones turísticas, sólo tienen razón de ser en la medida en que el turista se interese por la naturaleza, los atractivos culturales, etc., es decir por los elementos que generan la intención de llegar y permanecer en un lugar. Por lo tanto, sería poco sensato que los empresarios del ámbito turístico en general y de este sector hotelero en particular, no contribuyeran con la 
preservación y el equilibrio del ambiente y la sociedad local. La gestión ambiental contribuye a mejorar la gestión general de un establecimiento hotelero, al incorporar prácticas que generan impactos positivos en el entorno y nuevos mercados.

Los hoteles de ciudad constituyen una fuente de consumo de cantidades considerables de energía, agua, alimentos, entre otros recursos; y pueden emitir cantidades de contaminantes en términos de residuos sólidos, aguas residuales, humo, olor, ruidos y algunas sustancias químicas. Así, las instalaciones en su conjunto, son grandes generadoras de residuos sólidos, un consumidor ineficiente de agua y combustibles fósiles, grandes consumidores de papel y de una buena cantidad de productos no amigables con el medio ambiente, esto convoca a crear valores ambientales que influyan positivamente en sus actividades y su entorno (Moreno, 2006).

Los hoteles de ciudad disponen en la actualidad de diversos instrumentos y herramientas de gestión ambiental, una de ellas es la implementación de buenas prácticas ambientales hoteleras. La aplicación de buenas prácticas es una opción que se puede tener en cuenta por la contribución que las mismas pudieran brindar para la toma de conciencia y la mejora de los hábitos de los trabajadores y proveedores; además en su aplicación incorporar también a los clientes a través de actuaciones sencillas; lo que representará finalmente una reducción de los impactos ambientales negativos, un ahorro económico y una mejora de la imagen ambientalista para la instalación turística.

España, como uno de los países más destacados en la experiencia de la gestión ambiental en hoteles, posee diversos casos cuyos autores plantean metodologías y procedimientos asociados a la evaluación de la gestión ambiental en hoteles de ciudad. Así, Jiménez (2004) realizó un análisis de las dimensiones de la gestión ambiental en los servicios hoteleros. Al comienzo del estudio se realizaron varias entrevistas personales con directores de hotel, agencias de viaje y asociaciones hoteleras al objeto de conocer con más detalle el funcionamiento de los hoteles, sus prácticas ambientales y su terminología. Se abarcaron dos dimensiones principales: la gestión ambiental de los hoteles, así como la existencia de objetivos y plan ambiental.

Molina Azorín (2009), en su análisis de Sistemas de Gestión Ambiental, diseño organizativo y desempeño, con base en la industria hotelera española, planteó una serie de variables, entre las que se encuentran: la certificación medioambiental, diseño organizativo, rendimiento medioambiental y desempeño del hotel. Junto con el análisis de las anteriores variables y las relaciones entre la certificación medioambiental con el resto de las variables, también se ha analizado la relación entre la certificación medioambiental y tres características relevantes de los hoteles: la categoría del hotel (número de estrellas), tamaño (número de habitaciones y plazas) y el tipo de gestión (independiente o perteneciente a una cadena).

Sarriegi (2011), realizó un estudio de la evolución de la gestión ambiental en empresas industriales, específicamente en hoteles de la ciudad de Sevilla, para lo cual utilizaron la metodología Group Model Building (GMB). Esta metodología se caracteriza porque permite capturar información de un grupo de personas que son expertas en el problema a 
tratar. Para ello es necesario identificar y disponer de expertos que pueden aportar su conocimiento desde distintos puntos de vista. En este caso se seleccionaron personas expertas en la gestión ambiental de distintas empresas turísticas, consultorías ambientales y académicos con experiencia en proyectos ambientales. Para recopilar esta información se realizaron una serie de talleres en los que se reunieron los expertos. A la hora de recoger la información planteada por los expertos, la metodología GMB utiliza diferentes ejercicios. En el primer ejercicio, se identifican los agentes que intervienen en la gestión ambiental y se clasifican según el interés y la influencia en el problema investigado. En el segundo, se identifican las políticas que pueden afectar a la evolución de la gestión ambiental. En el tercero, se establecen los indicadores asociados a las distintas políticas identificadas y los resultados que pueden obtenerse. En el último ejercicio, los grupos de expertos elaboran las gráficas asociadas a los indicadores sobre las posibles evoluciones que pueden seguir los mismos según va madurando la gestión ambiental de la empresa.

Por su parte, Huamán (2015), en su investigación recogió información a partir de un cuestionario entregado a personas con responsabilidad en la gestión de los establecimientos hoteleros. El cuestionario incluye preguntas sobre cuestiones como la gestión y compra de productos ecológicos, la gestión de agua y energía, la formación ambiental impartida al personal, la participación del cliente en temas ambientales, o la implantación de algún sistema de gestión ambiental. El análisis realizado le permitió observar en qué medida se implantan determinadas prácticas medioambientales, además de valorar la percepción de las personas que responden sobre la gestión del medioambiente en establecimientos hoteleros.

En el caso de América, E. L. Z. y. C. V. Vargas (2011), evaluó la gestión ambiental del sector hotelero en el Destino mexicano de Cancún mediante un sistema de indicadores articulados en cuatro dimensiones: acciones de gestión ambiental, comunicación y consumo responsable, disposición a cumplir y monitoreo. Se destaca la experiencia de Ambiente (2015), que realizó la descripción del Programa de Manejo Ambiental o Sistema de Gestión Ambiental del hotel María José, en la costa del Pacífico. Este estudio permitió la caracterización de la instalación desde un enfoque medioambiental, así como determinar las brechas existentes para la aplicación de medidas correctivas.

Alonso Tamayo (2011), planteó en el análisis de un hotel en Cuba, que existe consenso al agrupar los aspectos ambientales de los servicios hoteleros de acuerdo a los elementos del entorno con los que interactúan. Entre ellos, son señalados el agua (consumo y vertidos), el suelo (residuos sólidos y residuos peligrosos), la atmósfera (consumo de energía y combustibles, emisiones de gases, ruidos y vibraciones) y los asociados a la ocupación del espacio, entre ellos, el uso del suelo y los efectos sobre la diversidad biológica.

Los casos anteriores demuestran el papel primordial que juega la gestión ambiental en las instalaciones hoteleras, y dentro de estas en los hoteles de ciudad, y su contribución al éxito del hotel. Actualmente se han implementado medidas para erradicar los problemas existentes en los hoteles de ciudad de diversas latitudes, sin embargo, muchos de ellos 
persisten por el carácter sistémico de la gestión ambiental en el planeta, lo que exige trabajar aún más no solo en el cuidado y conservación del medio ambiente, sino también en la generalización de buenas prácticas y en la profundización de su concepción teórica y práctica.

En los últimos 10 años han sido desarrolladas diversas investigaciones sobre la gestión ambiental en instalaciones hoteleras cubanas, cuyos aportes fueron de gran importancia para el desempeño ambiental y el futuro de dichas entidades. El análisis de casos de estudio relacionados con la aplicación de procedimientos para la gestión ambiental en hoteles en las tres regiones de Cuba (Campbell Margenat (2009), Lorenzo Linares (2011), Paz Ramírez (2015), Azze Alonso (2016) y Martínez Rodríguez (2017)), permitió resumir la coincidencia en la selección de indicadores representativos de la gestión ambiental como herramienta necesaria para la evaluación y diagnóstico ambiental de la instalación hotelera, estos se muestran en la figura 1.

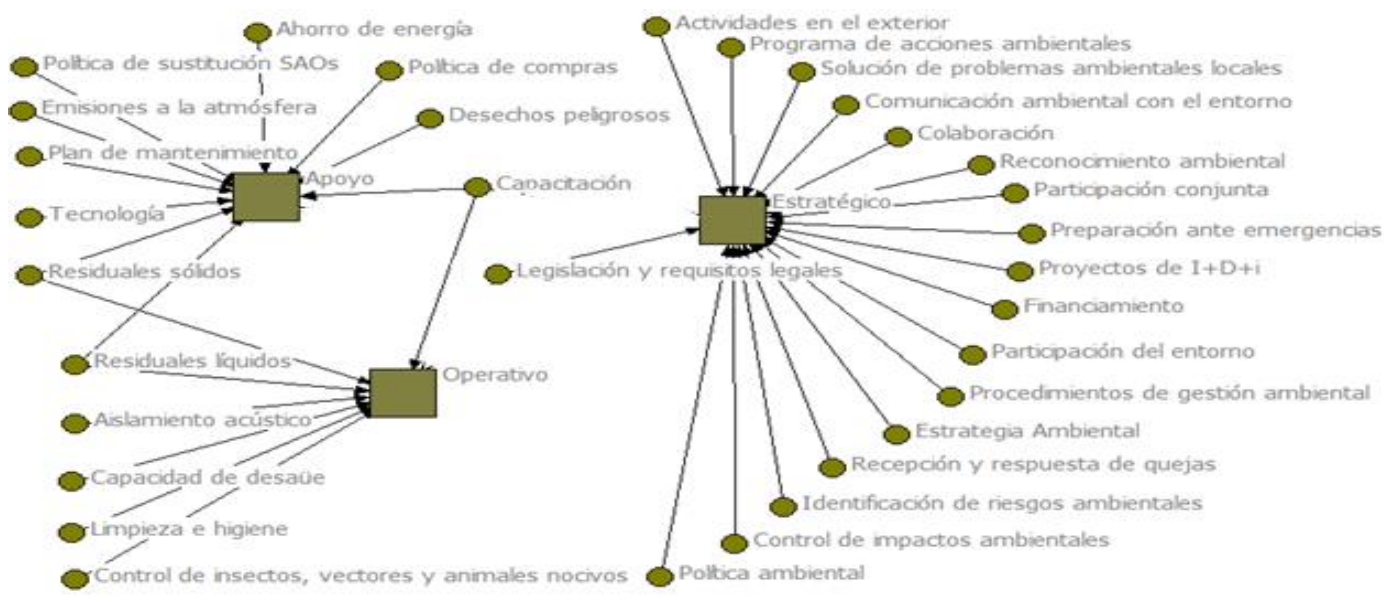

Figura 1. Indicadores por componente

Fuente: Elaboración propia

En virtud de lo anterior y al retomar la concepción de la gestión ambiental por componentes aportada por Reyes Chapman (2018), resulta fundamental la contextualización de la gestión ambiental a los procesos en los hoteles. Para el caso de un hotel de ciudad, se considera de gran importancia las interrelaciones de los componentes del mapa de procesos con los elementos del medio ambiente interno y externo, para lo cual es necesario proponer indicadores representativos de cada componente, en correspondencia con el tipo de instalación hotelera.

El estudio de las instalaciones hoteleras y las metodologías referidas permitieron sintetizar la pertinencia de evaluar los componentes de la gestión ambiental en hoteles mediante indicadores representativos, así cada uno incluiría, por ejemplo:

- Componente estratégico: Política ambiental, objetivos ambientales, legislación ambiental y otros requisitos legales, liderazgo y compromiso de la alta dirección, capacitación de los actores internos, responsabilidades y roles ambientales, evaluación de aspectos e impactos ambientales, disponibilidad de financiamiento, riesgos y oportunidades, programa de acciones ambientales, proyectos 
ambientales de $\mathrm{I}+\mathrm{d}+\mathrm{i}$ e identificación de situaciones potenciales de emergencia, tecnología.

- Componente operativo: Condiciones higiénico-sanitarias, mantenimiento y cuidado, sistema de tratamiento de residuales líquidos, regulaciones vigentes, entorno, capacitación del personal, control de vectores y animales nocivos, ubicación, capacidad de desagüe, desechos sólidos y aislamiento acústico.

- Componente de apoyo: uso racional del agua, energía y combustible, procedimiento de compras, manejo integral de desechos peligros, ambiente laboral, emisiones a la atmósfera (ruido, vibraciones, sustancia agotadora capa ozono y calidad del aire), contaminación (residuales líquidos, residuales sólidos, desechos peligrosos) y contratación con entidades para disposición final de los residuos.

A modo de síntesis, se considera que la gestión ambiental en hoteles de ciudad, caracterizada por el análisis de componentes e indicadores en los procesos, es una forma integrada de contribuir a la mejora del medio ambiente del hotel, y esta debe constituir un elemento de motivación tanto para los clientes internos como externos, además resulta oportuno el aporte de nuevas herramientas que mejoren los resultados de la gestión ambiental hotelera por su incidencia directa en el desarrollo del sector turístico.

\section{Aproximación a la gestión ambiental en el hotel Iberostar Ordoño en Gibara}

En este epígrafe se realiza un acercamiento a la gestión ambiental en un hotel de ciudad, en específico en el municipio de Gibara, perteneciente al Grupo Hotelero Cubanacán, el cual fomenta el cuidado del medio ambiente en sus instalaciones hoteleras. Este Grupo posee una encuesta para la actualización ambiental en sus hoteles, pero está dirigida solamente a la modalidad de sol y playa, lo cual no la hace aplicable a todas las instalaciones del grupo, además de ser insuficiente con respecto a los indicadores ambientales que evalúa.

El Complejo Hotelero Iberostar Gibara (CHIG), perteneciente a la cadena Iberostar Hotels \& Resorts, pionera del turismo sostenible, y al Grupo hotelero Cubanacán adoptó una posición de respeto al medio ambiente al definir su misión y visión. El CHIG tiene como misión: ofrecer experiencias vacacionales que dejan huella, en las mejores ubicaciones del mundo y con un modelo de turismo sostenible con respeto por las personas y el medioambiente. Su visión es: ser un referente internacional en turismo sostenible, con un modelo de negocio 100\% familiar, rentable y sólido, líder en calidad y satisfacción de nuestros clientes, basado en una cultura organizativa abierta que fomenta la innovación y el trabajo en equipo.

Como parte de este complejo hotelero, el Hotel Iberostar Ordoño está ubicado en la calle J. Peralta, entre Donato Mármol e Independencia, en pleno centro histórico de la ciudad de Gibara, a solo 100 metros del Parque Calixto García y la iglesia San Fulgencio y cercano a los museos, parques y otras instalaciones histórico-recreativo-culturales. El Ordoño, construido en el año 1927 es un edificio de estilo ecléctico cuyo dueño fue el 
comerciante asturiano Felipe Ordoño Díaz. Su decoración armoniza con la época en que fue construido, predominan los colores cálidos y suaves en paredes y techos.

El hotel se inauguró en enero de 2013, bajo la marca Encanto, y fue el primero de los que conformaron la Empresa Integral Turística de Gibara, además forma parte de un proyecto de desarrollo de Gibara como municipio turístico, que se sustenta en los atractivos y potencialidades de la localidad para satisfacer las expectativas de los clientes que buscan el descanso en un entorno que asegura al visitante experiencias memorables.

A partir del estudio de las normas cubanas 126 del 2001 y 127 del 2014, así como del Decreto 281 de 2007 se procedió al análisis de la gestión ambiental en los procesos del hotel, lo que permitió definir el mapa de procesos de la instalación como se muestra en la figura 2, en la que se observa la presencia del responsable de medio ambiente en los procesos estratégicos, siendo un factor positivo a tener en cuenta. El componente operativo está compuesto por los procesos de alojamiento y restauración, los cuales tienen contacto directo con el cliente, al dar respuesta a sus exigencias y contribuir a su satisfacción. Por último, se destaca el componente de apoyo, constituido por los procesos de gestión de recursos humanos y operación.

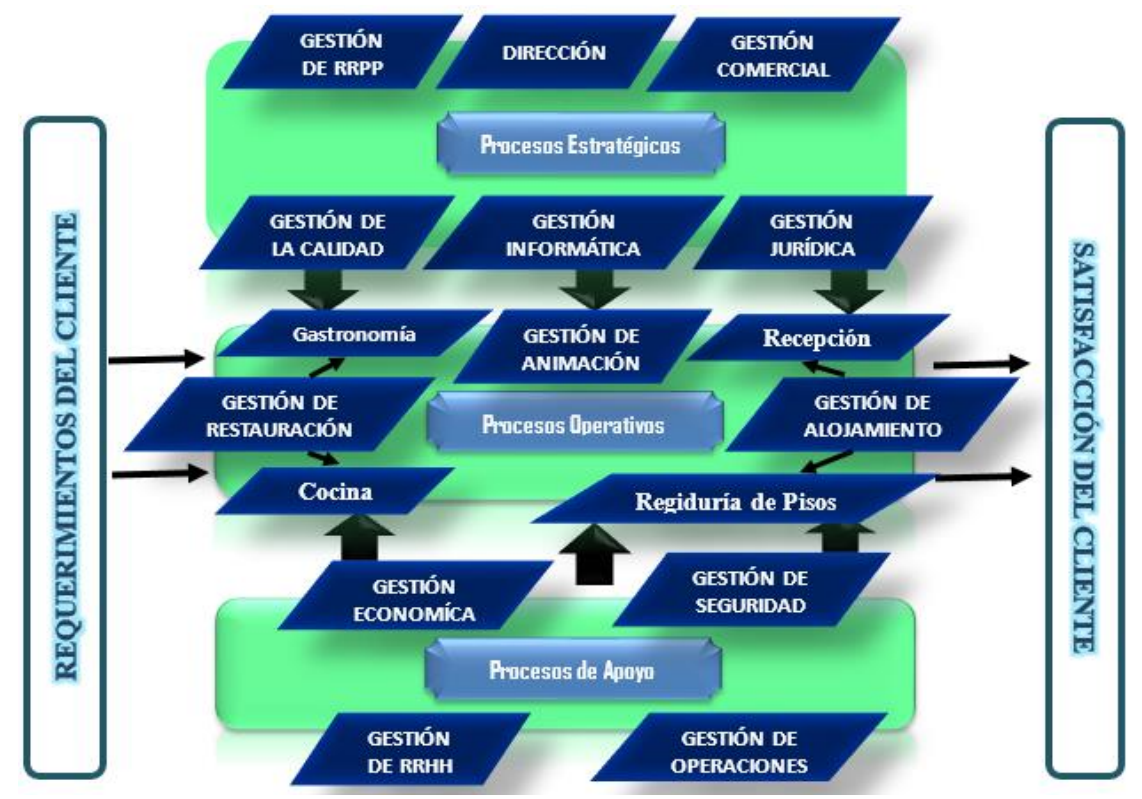

Figura 2. Mapa de procesos del hotel Iberostar Ordoño

Fuente: Elaboración propia a partir de documentación del Hotel Iberostar Gibara (2018)

El Hotel Iberostar Ordoño posee una planta de tratamiento de residuales, de esta manera los residuales son dispuestos, se deshidratan y se minimiza su impacto al no verter residuos sin tratar directamente al medio ambiente. La instalación cuenta además con un sistema de paneles solares, los cuales proporcionan energía eléctrica de una manera sostenible. En sus inicios, el hotel no se caracterizó por su especial atención a los temas medioambientales, solo se regía por determinados aspectos establecidos en algunas legislaciones vigentes, al no contar con un responsable de esta labor a nivel de hotel. 
Además, la instalación carecía de una estrategia ambiental propia, solo existía la estrategia ambiental a nivel de empresa en general.

Actualmente la cadena Iberostar Hotels \& Resorts ha fomentado el turismo sostenible en sus instalaciones, por lo que se han realizado cambios en la gestión ambiental que mejoran su desempeño. Así, se estableció el cargo de responsable del medio ambiente y sus funciones, además fue actualizada gran parte de la documentación asociada a los aspectos legales, normas y reconocimientos ambientales. La revisión ambiental del Hotel Iberostar Ordoño realizada en el año 2018 arrojó que las principales fuentes de generación de residuales líquidos son los procesos de alojamiento, elaboración y consumo de alimentos y bebidas, así como la limpieza de las instalaciones. En resumen, las insuficiencias prácticas identificadas fueron:

1. Vertimiento de residuales líquidos directamente al ecosistema sin tratamiento adecuado, así como contaminación atmosférica por emisiones de gases y ruido, lo que provoca insatisfacciones y quejas

2. Carencia de financiamiento para el despliegue de acciones ambientales que respondan a problemáticas identificadas, lo que limita el cumplimiento de la legislación vigente

3. Insuficiente conocimiento en trabajadores en general sobre la gestión ambiental en hoteles como proceso trasversal al resto de los procesos.

Si bien se realizan acciones para eliminar los problemas identificados, estos aún no han sido erradicados, lo que justifica la significación de la investigación para dar respuesta a las insuficiencias en la gestión ambiental, que limitan la mejora del medio ambiente en el Hotel Iberostar Ordoño, problema definido en la investigación.

\section{Mejoras en la gestión ambiental del hotel Iberostar Ordoño}

Las reformas en la gestión ambiental del hotel Iberostar Ordoño son el resultado de la aplicación del procedimiento para la gestión ambiental integral que se muestra en la figura 2 , el cual constituye una herramienta de intervención profesional a utilizar por los trabajadores del hotel y contempla cada uno de los elementos que propician el desarrollo de acciones de diagnóstico y mejora, desde los procesos de la organización a partir de los componentes estratégico, operativo y de apoyo, lo cual posibilita que la alta dirección se concentre en la evaluación, estructuración y gestión de las mejoras con perspectiva integradora. 


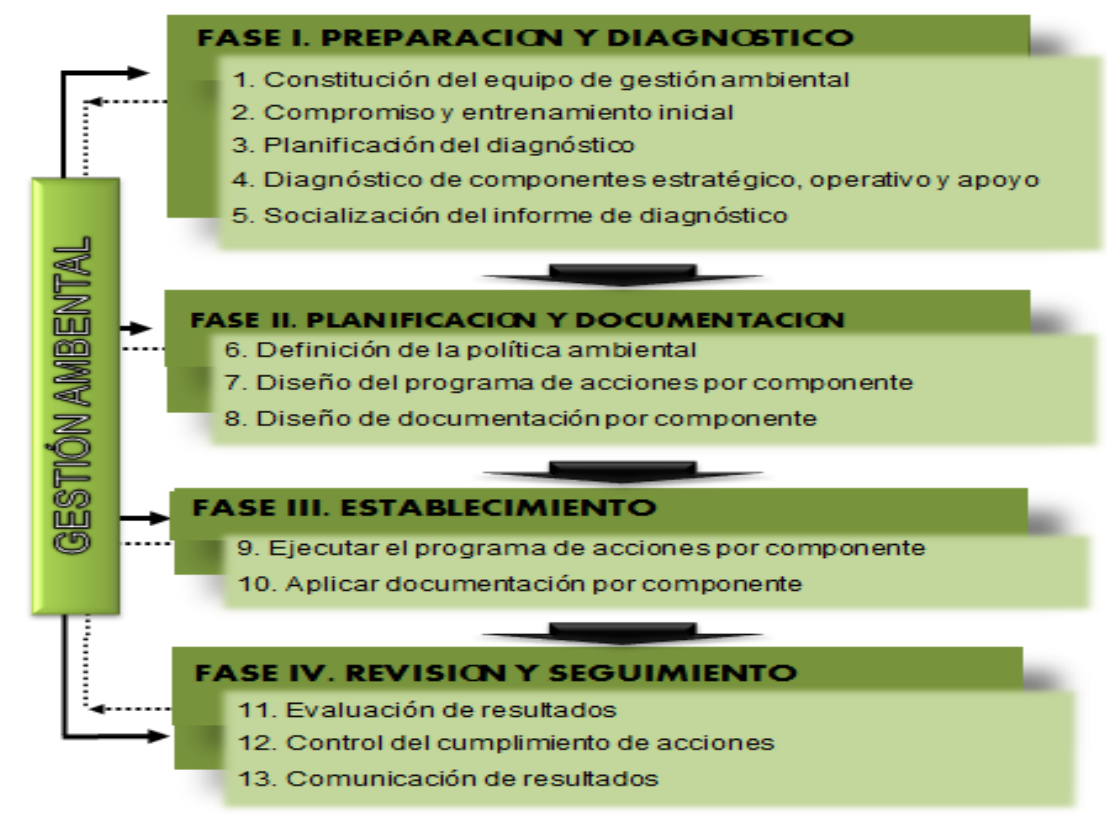

Figura 2 Procedimiento general para la gestión ambiental integral

Fuente: Elaboración propia a partir de Ochoa Avila (2015) y Reyes Chapman (2018)

El procedimiento se fundamenta en Ciclo Deming o PHVA (planear, hacer, verificar y actuar), que permite la mejora responsable del medio ambiente organizacional. El carácter cíclico e iterativo en el mismo se establece por la necesidad de retornar a la planificación y evaluar los resultados obtenidos que deben ser superiores a los que existían antes del comienzo. El funcionamiento y la interrelación sistemática de los componentes del sistema favorecerán el desempeño ambiental de la entidad. Los resultados fundamentales de la aplicación del procedimiento en el hotel Iberostar Ordoño se explicitan a continuación.

\section{Fase I: Preparación y diagnóstico}

Se crearon las condiciones de partida en la instalación hotelera para la identificación de los problemas asociados a la gestión ambiental, y la determinación de las oportunidades de acción para la implantación de este proceso de forma integral.

\section{Paso 1. Constitución del Equipo Gestor Ambiental}

Se conformó el Equipo Gestor Ambiental (EGA) del Hotel Iberostar Ordoño, compuesto por: Especialista en Animación y Medio Ambiente, Asesor Jurídico, Especialista en Gestión Económica, Especialista de Mantenimiento, Especialista de Recursos Humanos, Ama de Llaves, Especialista de Relaciones Públicas, Maître y Chef. En reunión efectuada y regida por la dirección de la instalación se eligió además el cargo de Representante de la Gestión Ambiental que es el responsable de liderar la actividad y representarla ante el Consejo de Dirección, el cual recayó en el especialista de animación y medio ambiente. Fue definida su responsabilidad, autoridad y aseguramiento para la aplicación del Procedimiento para la gestión ambiental integral en el hotel.

\section{Paso 2. Compromiso y entrenamiento inicial}


Se impartieron tres capacitaciones al EGA por parte del representante de gestión ambiental para lograr una familiarización y compromiso con los aspectos del diagnóstico a realizar, ya que estos serán los encargados de esta actividad. En estas actividades de capacitación se hizo énfasis en la importancia del diagnóstico, sus herramientas, las ventajas de favorecer la gestión ambiental desde una concepción integral en la instalación, así como las consecuencias que traería un incorrecto cuidado medioambiental, y la importancia del compromiso del equipo de realizar con calidad y profundidad el diagnóstico.

\section{Paso 3. Planificación del diagnóstico}

La relevancia del proceso de gestión ambiental fue abordada en reuniones realizadas, así como la importancia de la realización del diagnóstico de los principales aspectos ambientales de cada proceso, y de esa manera crear las bases para el diseño e implantación del procedimiento para la búsqueda de soluciones a los problemas ambientales a identificar. Se acordó en el Consejo de Dirección que el diagnóstico ambiental se debía realizar en un plazo de dos meses, alineado a los procesos del hotel, a la política general y dirección estratégica. Se establecieron los objetivos, las metas, las fechas de cumplimiento, los participantes, recursos necesarios y responsables.

\section{Paso 4. Diagnóstico del estado de los componentes de la gestión ambiental}

La gestión ambiental en el Hotel Ordoño se caracteriza por su estrecha interrelación con el ecosistema costero, interactúa además con el espacio citadino y la complejidad de su infraestructura, compuesta por empresas, instituciones, viviendas, por lo que el medio ambiente interno se encuentra muy interconectado al medio ambiente externo (fig.3).

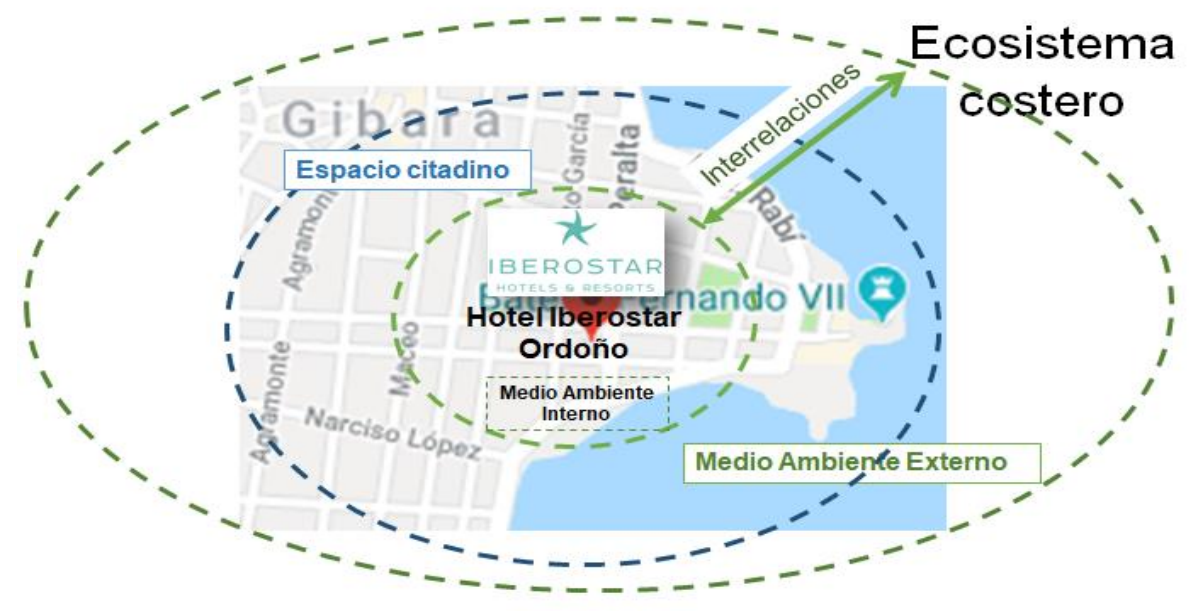

Figura 3. Interrelaciones entre el hotel y los subsistemas de la gestión ambiental

Fuente: elaboración propia

Para el análisis del estado de la gestión ambiental integral en el hotel Iberostar Ordoño se contó con el liderazgo del EGA. Fueron identificados una serie de problemas generales, los cuales fueron detectados a través del análisis realizado de cada componente mediante 
la aplicación de instrumentos propuestos, para lo que se tomó una muestra de 23 trabajadores, que incluyó al Consejo de Dirección y los jefes de procesos.

El comportamiento del componente estratégico interno arrojó que más del 75\% de los encuestados tienen conocimiento acerca de la existencia de una Estrategia Ambiental propia de la instalación, así como de la Política Ambiental, aunque esta no es suficientemente comunicada al entorno, así como los requisitos legales que debe cumplir la organización. Se demostró la existencia de procedimientos específicos sobre cómo preparase y responder a situaciones potenciales de emergencia. Por otra parte, se corrobora la inexistencia de financiamiento para la puesta en práctica de las acciones propuestas en el programa de acciones, la escasa participación en proyectos ambientales de I+D+i. Además, se debe trabajar en impulsar la capacitación del personal y que incluya temas que puedan sensibilizar y cambiar las conductas a seguir para causar impactos ambientales positivos que sean significativos, de manera que se evidencie su competencia sobre la base de la educación, capacitación y experiencia ambiental apropiada.

La evaluación del componente estratégico externo obtuvo como resultado que más del $70 \%$ de los encuestados afirmó que han sido identificados los riesgos ambientales de la instalación, así como la existencia de reconocimientos ambientales que fueron socializados a través de reuniones del Representante de la Gestión Ambiental con los trabajadores, pero existen deficiencias en la comunicación y la realización de acciones que involucren el entorno externo.

El componente operativo arrojó que más del $80 \%$ consideró positiva la limpieza e higiene en las actividades, productos y servicios del hotel, el tratamiento brindado a los residuales sólidos, así como un adecuado nivel de ruido. La capacitación fue un aspecto considerado como deficiente, además del tratamiento a los residuales líquidos, debido a la inestabilidad en el funcionamiento de la planta de tratamiento de residuales.

Con respecto al comportamiento del componente de apoyo se obtuvo que se cuenta con programas de ahorro de energía, de agua, y de residuos sólidos y líquidos, los cuales son chequeados sistemáticamente en el Consejo de Dirección, pero los mismos no han sido actualizados tras haber ocurrido el cambio de subordinación del hotel. En resumen, el diagnóstico permitió identificar dificultades en la política de compras para la adquisición de tecnologías, productos, materias primas e insumos no contaminantes con el medio ambiente; la mayoría de los trabajadores manifestaron la falta de capacitación como un aspecto a considerar de relevancia. Se muestran deficiencias respecto al plan de mantenimiento y reparaciones, por lo que no se realizan revisiones periódicas a los equipos informáticos, refrigeración y transporte. La tecnología existente, a pesar de ser moderna, ha ocasionado una serie de problemas, un ejemplo de ello lo constituye la planta de tratamiento de residuales, la cual necesita ser remplazada cada dos años, situación que se ha visto imposibilitada por la carencia de presupuesto, esto genera dificultades con el tratamiento a los residuales líquidos.

Paso 5. Socialización del informe final del diagnóstico 
El informe del diagnóstico fue elaborado a partir de los datos recogidos por el EGA y posteriormente fue presentado al Consejo de Dirección, el cual tuvo una información precisa de las principales dificultades y de las necesidades para transformar la situación ambiental de la instalación, con posterioridad se hizo la presentación del informe por el EGA a los trabajadores, los cuales fueron actualizados sobre los principales problemas detectados en cada componente y las acciones que darían tratamiento a los mismos.

\section{Fase II: Planificación y documentación}

Se establecieron los elementos para la planificación de la gestión ambiental integral a realizarse por la dirección del hotel, a través del diseño de objetivos estratégicos los cuales se incorporaron a un programa de acciones ambientales por componente estratégico, operativo y de apoyo para su cumplimiento.

\section{Paso 6. Definición de la política ambiental}

La Política Ambiental fue actualizada y se definió como: "El Hotel Iberostar Ordoño cuyo objeto social está dirigido a la satisfacción del cliente nacional y extranjero, reconoce su responsabilidad en la preservación del Medio Ambiente".

Como parte de su Política Ambiental, el Consejo de Dirección y sus trabajadores se compromete a desarrollar una actividad que permita obtener resultados satisfactorios con la optimización de las tecnologías existentes, para el logro de un desarrollo sostenible en correspondencia con la Estrategia Ambiental Nacional, teniendo en cuenta el cumplimiento de las leyes y regulaciones vigentes en nuestro país en gestión ambiental. Además, involucrando a todo el personal de la organización mediante la capacitación, y al establecer como premisa la mejora responsable de la gestión ambiental, a través del uso adecuado de las materias primas e insumos utilizados para mitigar al máximo los impactos negativos en el medio ambiente.

Los objetivos estratégicos definidos fueron:

1. Cumplir con las Legislaciones vigentes aplicables al hotel en materia de Medio ambiente

2. Capacitar, documentar e instruir a los trabajadores en temas relacionados con la protección del medio ambiente

3. Lograr la mejora continua el desempeño ambiental en los servicios

4. Reducir el sobre consumo de productos y materias primas

5. Reducir los impactos ambientales negativos en los procesos

6. Favorecer la transversalidad de la gestión ambiental en todos los procesos del hotel.

\section{Paso 7. Diseño del programa de acciones por componente}

Se diseñó un Programa de gestión ambiental por componente estratégico, operativo, y de apoyo, para el período 2019-2020, este recoge 22 acciones a tener en cuenta para contribuir a la solución de los problemas identificados y a la mejora del medio ambiente en el Hotel Iberostar Ordoño. Las acciones responden a los objetivos de la Agenda 2030 
de la ONU y al Plan de Estado Tarea Vida de Cuba. En el cuadro 1 se muestra un resumen del programa.

Tabla 1. Resumen del Programa de gestión ambiental del Hotel Iberostar Ordoño

\section{Componente de la gestión ambiental}

Acción

$\begin{array}{cc}\text { Agenda } & \text { Tarea } \\ \text { ONU 2030 } & \text { Vida }\end{array}$

\begin{tabular}{llll}
\hline Estratégico & $\begin{array}{l}\text { Actualizar la } \\
\text { documentación ambiental } \\
\text { de los procesos }\end{array}$ & Objetivo 4.7 & $\begin{array}{l}\text { Tareas 10 } \\
\text { y } 11\end{array}$ \\
& $\begin{array}{l}\text { Diseñar proyectos de I+D+i } \\
\text { asociados a la gestión }\end{array}$ & Objetivo 11 & \\
ambiental a corto y & & \\
& mediano plazo para adquirir & & \\
& financiamiento & & \\
& Desarrollar talleres & Objetivo & \\
& ambientales con la & 11.3 & \\
& comunidad para la toma de & & \\
& conciencia, en el marco de & & \\
& eventos de gran relevancia & & \\
para el Destino, como "El & & \\
& Festival Internacional de & & \\
& Cine de Gibara" & &
\end{tabular}

Operativo

Analizar con el MINTUR la

Objetivos 12 Tarea 11 adquisición de nuevas y 14.7 tecnologías aplicables a los procesos del hotel, que no dependan de grandes inversiones Garantizar un adecuado Objetivo 8.4 Tarea 4 manejo de los residuos sólidos que se generan en la instalación (recolección, almacenamiento y transportación), clasificándose en recuperables y no recuperables 
Capacitar a los trabajadores Objetivo 4.7

de los procesos operativos

sobre buenas prácticas

ambientales en hoteles

\begin{tabular}{|c|c|c|c|}
\hline \multirow[t]{3}{*}{ Apoyo } & $\begin{array}{l}\text { Actualizar el programa de } \\
\text { ahorro de agua }\end{array}$ & Objetivo 6.3 & Tarea 4 \\
\hline & $\begin{array}{l}\text { Actualizar el programa de } \\
\text { ahorro de energía }\end{array}$ & Objetivo $7 b$ & Tarea 8 \\
\hline & $\begin{array}{l}\text { Establecer una política de } \\
\text { compras para la adquisición } \\
\text { de tecnologías, productos, } \\
\text { materias primas e insumos } \\
\text { amigables con el medio } \\
\text { ambiente }\end{array}$ & Objetivo 8.2 & \\
\hline
\end{tabular}

Para la valoración de la aplicación del procedimiento fueron seleccionados 13 especialistas: cuatro pertenecientes a la Delegación del Ministerio de Ciencia Tecnología y Medio Ambiente (CITMA), y los nueve restantes vinculados a otras instalaciones del CHIG. De ellos, siete poseían un nivel de conocimiento alto y seis de nivel medio, por lo que estos últimos fueron descartados. A los siete especialistas seleccionados se les aplicó un test para garantizar la retroalimentación necesaria con respecto a la evaluación del Procedimiento de gestión ambiental integral basado en componente estratégico, operativo y de apoyo, además de los indicadores respectivos propuestos.

A cada elemento se le otorgó una calificación del 1 al 5 (escala de Likert), donde 1 es el menor valor y 5 el de mayor valor. Para la evaluación de las fases y pasos del procedimiento, así como de los aspectos propuestos para evaluar cada componente. Para la primera fase del procedimiento el índice de evaluación fue de 4.71, la segunda fase obtuvo un índice de evaluación de 4.68, la evaluación de la tercera fase fue de 4.90, y la cuarta fase obtuvo un índice de satisfacción de 4.67, lo que significa que las cuatro fases fueron evaluadas de excelente por los especialistas.

Con respecto a los aspectos propuestos para evaluar cada componente, estos fueron también evaluados de excelente, donde los pertenecientes al componente estratégico obtuvieron un índice de evaluación de 4.66, el índice de los indicadores del componente operativo fue de 4.83 y el índice del componente de apoyo obtuvo 4.87.

De forma general, el consenso logrado entre los especialistas acerca del procedimiento y los indicadores por componente, arroja una opinión grupal consensuada, lo que ofrece certeza de estabilidad en el procedimiento propuesto. Lo anterior demuestra la actualidad teórico-práctica del procedimiento propuesto; muestra su consistencia lógica visible; manifiesta que es comprensible y asimilable por los actores involucrados en los límites 
que establece su complejidad intrínseca; así como la posibilidad de su generalización en otras instalaciones turísticas del Destino Gibara o a nivel de país.

\section{Conclusiones}

- El análisis del marco teórico-práctico referencial demuestra que no se aborda suficientemente la gestión ambiental desde una visión integral en hoteles, son escasas las investigaciones sobre las dimensiones y variables de la gestión ambiental en hoteles de ciudad, no se encontraron suficientes precedentes de tratamiento a los componentes estratégico, operativo y de apoyo en hoteles, lo que evidencia la gran actualidad y pertinencia del tema investigado.

- Se diseñó un Procedimiento para la gestión ambiental integral para el Hotel Iberostar Ordoño, compuesto por cuatro fases y 13 pasos, que posee las potencialidades necesarias para asegurar su coherencia, pertinencia e impacto favorable, y crear las bases para el diseño de una solución al problema, por cuanto permitió caracterizar, intervenir y mejorar la gestión ambiental en el campo práctico.

- La aplicación parcial hasta la fase II del Procedimiento para la gestión ambiental integral en el Hotel Iberostar Ordoño, permitió diseñar un programa para la gestión ambiental que agrupa las acciones asociadas a los problemas identificados en los tres componentes, su cumplimiento puede transformar la situación ambiental actual en el hotel y contribuir al cumplimiento de objetivos del desarrollo sostenible 2030 y del Plan de Estado Tarea Vida de Cuba 2050.

- El criterio de los especialistas sobre el procedimiento permitió constatar su capacidad real para formular e implantar estrategias efectivas de mejora del medio ambiente del hotel, esencialmente al proponer al Consejo de Dirección las herramientas metodológicas tácticas y estratégicas para conducir el proceso de gestión ambiental, todo lo cual permite contribuir a la mejora del medio ambiente interno y del medio ambiente del Destino Gibara.

\section{Referencias bibliográficas}

126, N. C. (2001). Industria Turística - Requisitos para la clasificación por categorías de los restaurantes que prestan servicio al turismo.

127, N. C. (2014). Industria Turística - Requisitos para la clasificación por categorías de los establecimientos de alojamiento turístico.

281, D. (2007). Reglamento para la gestión de la dirección y sus subsistemas.

Alonso Tamayo, L. (2011). Procedimiento para el monitoreo ambiental en empresas hoteleras. Caso de estudio Hotel SunBeach. (Tesis en Opción al Título de Ingeniero Industrial), Universidad de Matanzas "Camilo Cienfuegos".

Ambiente, C. S. (2015). Declaración de Impacto Ambiental Expost y Plan de Manejo Ambiental del Hotel María José. 
Atencio Zayas, W. (2015). Procedimiento para el Diseño e Implantación del Sistema de Gestión Ambiental en la Empresa Inmobiliaria del Turismo de Holguín. (Tesis en opción al Grado Científico de Máster en Gestión Ambiental), Universidad de Holguín.

Azze Alonso, A. M. (2016). Procedimiento General Para la Gestión Ambiental en la Villa Mirador de Mayabe. Universidad de Holguín.

Calvo Herrrero, R. (2010). El Sistema Europeo de Gestión y Auditoría Ambiental (EMAS), una herramienta transversal para que las empresas mejoren su sostenibilidad ambiental.

Campbell Margenat, A. d. 1. A. (2009). Propuesta de un Programa de Gestión Ambiental en el Hotel Villa la Granjita Hostal Mascotte de Villa Clara. Universidad Central "Marta Abreus de las Villas"

González Abreu, B. (2015). Procedimiento General Para la Gestión Ambiental Integral en el Hotel Brisas Covarrubias de las Tunas. Universidad de Holguín.

Huamán, L., Soledad, Claudia (2015). Gestión ambiental en los hoteles de Santiago de Compostela.

Isaac Godínez, C. L. R. C., Roberto (2012). Manual de la gestión ambiental organizacional.

ISO-14001. (2015). Sistema de Gestión Ambiental-Requisitos con orientaciones para su uso.

Jiménez, J. C. y. J. d. B. (2004). Un análisis de las dimensiones de la gestión ambiental en los servicios hoteleros.

Lorenzo Linares, H. (2011). Implementación de un Sistema de Gestión Medioambiental. Caso Hotel NH Kristal Laguna Cayo Coco.

Martínez Rodríguez, M. (2017). Procedimiento para Sistemas de Gestión Ambiental en hoteles de Varadero Cuba.

Molina Azorín, J. F. (2009). Sistemas de Gestión Ambiental, diseño organizativo y desempeño un análisis de la industria hotelera española.

Moreno, E. (2006). La Política de calidad ante la nueva realidad del turismo y sus transformaciones.

Ochoa Ávila, M. B. (2014). Tecnología para la gestión ambiental integral en instituciones escolares. Aplicación en Holguín. (Tesis presentada en opción al Grado Científico de Doctora en Ciencias Técnicas). 
Paz Ramírez, Y. (2015). Procedimiento Metodológico Integrado para la Implementación de un Sistema de Gestión Ambiental en el Hotel Brisas Covarrubias.

Reyes Chapman, B. (2018). Procedimiento para la gestión ambiental en el Centro de Información y Gestión Tecnológica de Holguin. (Tesis presentada en opción al título académico de Máster en Gestión Ambiental), Universidad de Holguín.

Sarriegi, J. M. y. M. O. (2011). Estudio de la Evolución de la Gestión Ambiental en Empresas Industriales. Caso hoteles de la ciudad de Sevilla.

Vargas, E. L. Z. y. C. V. (2011). Turismo y ambiente. Un estudio de los comportamientos ambientales en el sector hotelero de Cancún (D. Suárez Ed.): Editorial Académica Española. 


\section{PARA CITAR EL ARTÍCULO INDEXADO.}

Barbarita Ochoa Avila, M., \& Leyva Driggs, D. (2021). Mejoras en la gestión ambiental del hotel Iberostar Ordoño del destino turístico de Gibara, Cuba. Explorador Digital, 5(1), 297-316. https://doi.org/10.33262/exploradordigital.v5i1.1504

\section{¿Ciencia}

El artículo que se publica es de exclusiva responsabilidad de los autores y no necesariamente reflejan el pensamiento de la Revista Explorador Digital.

$\mathrm{El}$ artículo queda en propiedad de la revista y, por tanto, su publicación parcial y/o total en otro medio tiene que ser autorizado por el director de la Revista Explorador Digital.
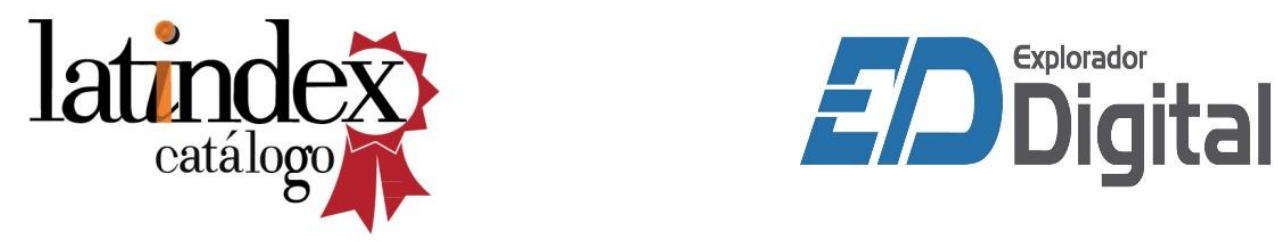